\title{
COMPORTAMIENTO DISCIPLINARIO EN LA ESCUELA “NIÑO JESÚS", SIUNA, REGIÓN AUTÓNOMA ATLÁNTICO NORTE, 2011
}

Rosa Francisca Montenegro Flores ${ }^{[1]}$ Reina Isabel Hernández Herrera ${ }^{[2]}$ Angélica Leonor Ruiz Calderón ${ }^{[3]}$

\section{Resumen}

El estudio se realizó en el Centro Escolar "Niño Jesús" del barrio "Dolores Marín”, sector urbano del municipio de Siuna, Región Autónoma Atlántico Norte (RAAN), a $334 \mathrm{~km}$ de la capital Managua. El objetivo fue determinar el comportamiento disciplinario con una población de estudio de 82 niñas y 103 niños del centro educativo, 7 docentes, 1 director, 78 padres y madres de familia. Destacándose que los factores sociales que han afectado el comportamiento disciplinario en la niñez es producto de la educación brindada desde el entorno familiar. En la etapa de la niñez, se está propenso a tener problemas conductuales, cuando se es afectado por la situación económica, su condición de vida, el estado emocional, la desintegración familiar y la pedagogía inadecuada; asimismo, el papel que ha desempeñado la Consejería Escolar en este contexto en la orientación e implementación de planes pedagógicos para resolver los diferentes problemas. La investigación fue cualitativa de carácter descriptivo. Las técnicas empleadas fueron: grupo focal, entrevista y observación directa en las aulas de clase.

Palabras claves: Comportamiento disciplinario, factores pedagógicos.

\section{Summary}

The study was conducted at the "Niño Jesus" School, located in the "Dolores Marin" neighborhood, urban sector of Siuna Municipality, North Atlantic Autonomous Region (RAAN), at a distance of $334 \mathrm{~km}$ from the capital Managua. The objective was to determine the disciplinary behavior with a study population of 82 girls and 103 boys of the school, 7 teachers, 1 director, and 78 fathers and mothers. We highlight that the social factors that have affected the disciplinary behavior in the childhood is a product of the education provided from the family environment.

\footnotetext{
[1] Licenciada en Pedagogía con mención en Educación. Apoyo Admón. URACCAN Las Minas. montenegroflores@ yahoo.com

${ }^{[2]}$ Licenciada en Pedagogía con mención en Educación. Docente Educación Media - Siuna sin correo.

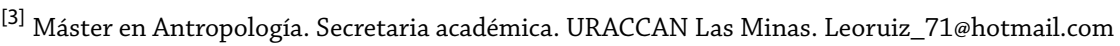


In the stage of childhood is likely to have behavioral problems, when someone is affected by the economic situation, their living conditions, emotional state, family disintegration and an inadequate pedagogy; also the role played by the School Counseling in this context is very important in the orientation and implementation of educational plans to solve different problems. The research was qualitative -descriptive. The techniques used were: focus group interview and direct observation in the classroom.

Keywords: Disciplinary behavior, pedagogical factors.

\section{Introducción}

A nivel mundial y específicamente en América Latina, la educación constituye uno de los ejes principales para el funcionamiento del proceso de enseñanza aprendizaje, enfrentando diferentes dificultades y uno de ellos es el comportamiento que se ha observado en las formas de actuar de la niñez en la mayoría de los centros escolares.

La educación en Nicaragua tiene como finalidad formar a la persona humana como un ser integral, capaz de actuar responsable y libremente en la sociedad, esa tarea implica una serie de actitudes, condiciones y capacidades que conllevan a un compromiso personal. En los centros escolares, los docentes se esfuerzan por orientar e implementar los valores morales, como el respeto a la vida y a las personas, la honestidad, responsabilidad y solidaridad. Que hoy en día es muy difícil porque el estudiantado se encapricha con los nuevos cambios como el poder, lo económico, el individualismo, el narcotráfico y la explotación sexual. Aun con esta dificultad la misión de los docentes y padres de familia es seguir luchando por el futuro de la niñez.

En la Región Autónoma Atlántico Norte, región multiétnica y pluricultural, de manera general existen diferencias educacionales por su composición urbana, y rural, el estudiantado se ve afectado por diversos comportamientos que inciden negativamente en el desarrollo integral, en los aprendizajes y la inserción individual de su medio familiar y social. Este problema de comportamiento en las escuelas afecta a la mayor parte de la población estudiantil, directores, docentes, madres y padres de familia.

En las escuelas del municipio de Siuna, el comportamiento es uno de los mayores problemas que se atraviesa, ya que la niñez ha sido objeto de conflictos para los adultos, a los cuales se les hace difícil poder ayudar a formar nuevos patrones de comportamiento en los centros escolares tantos urbanos como rurales, debido a que en muchas aulas de clases se encuentran entre 25 a 30 estudiantes de diferentes procedencias.

En los últimos años en la escuela "Niño Jesús" ubicada en el barrio "Dolores Marín", se presentan problemas de comportamiento disciplinario en la niñez, a los que se les 
han hecho llamados de atención verbal, provocando frustraciones y además una gran preocupación para docentes, padres y madres de familia e instituciones cuya labor es la educación. Desarrollando estrategias con el propósito de informarlos en temas relevantes para su desarrollo integral e interdisciplinario, auxiliados por psicólogas que capacitan a directores de centros escolares y dan seguimiento sistemático para alcanzar las metas de los Planes Pedagógicos, propuestos para la niñez que esta siendo atendida, promoviendo la auto evaluación, co-evaluación y evaluación del mismo con el apoyo del personal educativo.

\section{Revisión de literatura}

Cabassa (2006), mencionó que existen diferentes factores que determinan el comportamiento de la niñez. Algunos tienen que ver con las características individuales y su personalidad, el ambiente tanto del hogar como de la escuela. El docente que maneja la sala de clase, su compromiso con los estudiantes y su filosofía educativa puede darle un giro a los problemas que se enfrentan a diario en el salón.

\section{Factores socio pedagógicos en el comportamiento disciplinario de la niñez}

\section{Contexto social}

Es importante señalar que los factores sociales como publicidad, radio, televisión, ausencia de patrones conductuales adecuados, etc. Unidos al ambiente educativo se encuentran relacionados con los problemas de disciplina, del mismo modo y de forma directa con el entorno familiar y social (Moreno C.; 2001, p. 4).

\section{La desintegración familiar}

Este es uno de los problemas sociales más frecuentes en nuestro medio ya que la desintegración familiar o el divorcio, es algo que causa mucho sufrimiento, el dolor o la ira que puede sentir el padre o la madre como resultado de la separación, es transmitida a sus hijos e hijas (Rourat, 2001, p. 134).

\section{Padres y madre apáticos}

Los padres y madres de familia no pueden ignorar el mal comportamiento, sólo porque el niño y niña están en cierta edad, ni debido a su sistema familiar, tal actividad implica que los adultos son importantes para ayudar a los niños a formar nuevos patrones de conducta. (Cáceres C.; 2007, p. 4). 


\section{Factores pedagógicos}

\section{Factor escolar}

En la interacción profesor-estudiante surgen con frecuencia conflictos serios, así sucede cuando se da una actitud negativa del profesor que impide lograr un clima humano favorable, cuando enfatiza memorismo y no el aprendizaje significativo, o fomenta la competitividad y no la cooperación con los miembros del grupo (Beltrán J. ; 1995, p. 464).

\section{Pedagogía inadecuada}

Autores como Lambert \& Gardner identifican que una metodología inadecuada, proporciona un entorno de indisciplina dentro del aula de clase (Martínez, 2008: s/p).

\section{Influencia del factor económico en el comportamiento disciplinario}

El comportamiento está determinado según diversas causas socioeconómicas, incluye la falta de recursos del hogar para enfrentar los gastos que demanda la asistencia a la escuela (Flape, 2008, p. 17).

\section{Trabajo infantil}

Cuando se habla de trabajo infantil se refiere a cualquier actividad remunerada o no, que la niñez realiza, y que significa un peligro nocivo para su desarrollo físico, social, moral y psicológico, dicha actividad representa en la mayoría de los casos un obstáculo para su pleno desarrollo (Quintana, 2001, p. 6).

\section{Papel que desempeña la Consejería Escolar en relación al comportamiento}

En este sentido la Consejería Escolar juega un rol importante porque de esta forma se implementa un considerable trabajo de atención integral y especializada a estudiantes que están reflejando un comportamiento no adecuado, incidiendo así en las causas y consecuencias de estas prácticas o formas de violencia (De Trinidad, 2005, p. 17).

\section{Materiales y métodos}

La investigación se realizó en la escuela de Educación Primaria “Niño Jesús”, ubicada en el barrio “Dolores Marín”, Siuna, Región Autónoma Atlántico Norte, Nicaragua. El tipo de estudio es cualitativo y según el nivel de profundidad es descriptivo y refleja los sentimientos y percepciones de las personas observadas y entrevistadas. 
La población de estudio fue de 82 niñas, 103 niños del centro educativo, 7 docentes, 1 director, 78 madres y padres de familia y Consejería Escolar. Las técnicas empleadas fueron: grupo focal, entrevista y la observación directa en las aulas de clase.

\section{Resultados y discusión}

Existen diferentes factores que afectan el comportamiento de la niñez. Algunos tienen que ver con las características individuales de cada niño o niña, su personalidad. Otro factor es el ambiente, tanto del hogar como de la escuela. Tampoco podemos pasar por alto el factor maestro y el salón. La manera a través de la cual el profesor maneje la sala de clases, su compromiso con los estudiantes y su filosofía educativa pueden darle un giro a los problemas a los que se enfrenta a diario en el salón. Estos son algunos factores, existen muchos más.

\section{Factores sociopedagógicos en el comportamiento disciplinario de la niñez}

Los problemas de conducta en los centros escolares es una realidad que cada vez aparece con más intensidad y supone un alto nivel de preocupación de la comunidad educativa. El comportamiento perturbador que determinados estudiantes manifiestan no tienen significación por sí solo, se trata de un síntoma externalizado que esconde un conjunto de signos que no tienen por qué ser patológicos y que determinan dichas conductas. Su origen puede ser diferente para cada individuo a pesar de que el comportamiento sea en muchas ocasiones el mismo.

\section{Factores sociales}

\section{Contexto social}

Es relevante la manera en que percibe el estudiante su ambiente familiar y su dinámica. La importancia que sus padres le dan al estudio en casa, a las tareas en equipo, al tiempo que pasa en la escuela, a su percepción acerca de las capacidades y habilidades. El contexto familiar del estudiante determina los aspectos económicos, sociales y culturales que llegan a limitar o favorecer su desarrollo personal y educativo.

Para las docentes entrevistadas, el comportamiento de la niñez en estado de escolaridad ha venido cambiando, debido a la influencia de diferentes medios de recreación: televisión, radio y telefonía celular, lo que provoca comportamientos inadecuados y se aprecia en el aula de clases, porque no sólo vienen a recibir el pan de la enseñanza, sino que en su interactuación sus actitudes y su conducta es diferente.

Además, expresaron que en el comportamiento disciplinario intervienen las normas y reglas que se practican en los hogares, ya que ellos reflejan en el aula de clases lo que aprenden del entorno en el que se desenvuelven y se desarrollan. Es importante 
mencionar que muchos de ellos crecen en ambientes de violencia, agresiones, carencias, lo que repercute en el comportamiento de indisciplina escolar.

Lo que coincide con Moreno (2001), quien refiere que los factores sociales: publicidad, radio, televisión, ausencia de patrones conductuales adecuados, etc., unidos al ambiente educativo se encuentran relacionados con los problemas de disciplina, del mismo modo y de forma directa con el entorno familiar y social en el que se halla el estudiante, siendo este a su vez, el contexto donde se asientan las normas establecidas y se producen conductas de indisciplina; si se ignora el ambiente, tan sólo se estará obteniendo una visión parcial de lo que ocurre en el aula de clase.

Las docentes, en relación a los casos de indisciplina que se presentan en el centro educativo, han manifestado que se necesita más el apoyo de las madres y padres de familia, ya que son los actores principales en la formación de sus hijas e hijos, los cuales deben crecer en un ambiente lleno de amor, respeto y comprensión que les permita tener un desarrollo físico, emocional e intelectual para enfrentar la vida futura.

El comportamiento que presenta la niñez en la escuela se debe a la carencia afectiva de sus madres y padres, lo cual se caracteriza como familias conflictivas; el mal ejemplo que ven y escuchan a diario de otras personas con quienes se relacionan, es decir, el medio en que se desarrollan, desenvuelven y aprenden todos los días. El mundo cambia continuamente y con ello su forma de pensar y actuar, por lo que es necesario que se garanticen bases sólidas con valores y principios morales, además que tengan reglas y normas claras en el hogar que permitan una mejor formación.

\section{La desintegración familiar}

Incide en el comportamiento disciplinario de la niñez en edad escolar, cuyo problema es muy frecuente en este siglo. Cabe destacar que existen una variedad de situaciones que conducen a la pareja a tomar tal decisión, como infidelidad, parejas muy jóvenes, bajo nivel académico y en otros casos poca información sobre la formación de un hogar, afectando directamente el desarrollo de la niñez en el transcurso de su vida, lo que influiría a la hora de formar sus propias familias e incide de forma negativa en su vida académica tomando posturas inadecuadas de comportamiento dentro y fuera del aula de clase, opacando así su medio de socialización y creando en sí mismo una idea poco convincente de su futuro.

$\mathrm{Al}$ respecto Rourat (2001), señala que la desintegración familiar o el divorcio, es algo que les causa mucho sufrimiento; el dolor y la ira que puede sentir la madre o el padre, como resultado del divorcio, puede transmitirse a los hijos. La desintegración se asocia, en la niñez, con una incidencia mayor de comportamiento antisocial en el aula. Los estudiantes de familias divididas presentan comportamiento de indisciplina 


\section{EDUCACIÓN}

que dificulta el aprendizaje. Un hogar desintegrado puede provocar en el estudiante comportamientos inadecuados, tales como: inclinación a la drogadicción, prostitución, alcoholismo, etc., como puede suceder en el medio. Entre otros efectos pueden mencionarse: la falta de atención, agresividad y apatía, provocando malas relaciones entre sus compañeros.

En observaciones realizadas, nos dimos cuenta que la desintegración familiar incide en la conducta de la niñez, ya que la mayoría viven sólo con la mamá, abuela u otro pariente cercano, por lo tanto se sabe que si un niño vive solo con la mamá, este necesitará del amor de su papá, le hará analizar el daño que causa una separación para sus hijas e hijos. Tienen que ser reflexivos al momento de tomar decisiones que les ayude a reformar los hogares donde no les falte amor, dedicación y comunicación, de tal forma que les permita garantizar un futuro con posibilidades de triunfos en su vida emocional y profesional.

\section{Madres y padres apáticos}

Es evidente en el centro educativo la existencia de madres y padres apáticos con sus hijos, dejando en último plano la educación, y perjudicando de esta manera las relaciones familiares, conversaciones, juegos, intercambio de opiniones entre ellos, además se muestra la carencia de cariño y afecto mutuo cuando se tiene la oportunidad de hacerlo.

Una docente expresó:

El poco interés de algunos padres y madres de familia en ayudar a sus hijos genera que estos busquen otras formas de solventar sus problemas y necesidades desde pequeños, ejemplo de ello es que pasan mucho tiempo en la calle viendo y haciendo cosas incorrectas y es así como cambia el comportamiento e incluso pierden el amor, el respeto y la figura de sus progenitores.

Además, por el hecho de trabajar y cumplir con sus obligaciones laborales estos progenitores no permanecen en el hogar, y no tienen el tiempo para dedicarles a sus niños y niñas, para visitarlos en el centro educativo, no asisten a las reuniones, no se presentan a retirar boletines, no están al pendiente de preguntarle al docente cómo se ha estado portando, no existe esa relación, esa comunicación, no investigan el rendimiento escolar de sus criaturas, sin embargo hay muchos que tienen tiempo suficiente y que permanecen en el hogar, y de igual manera no participan en las actividades escolares, porque son irresponsables con la educación de sus hijas e hijos.

Para Cáceres (2007), los padres y madres de familia no pueden ignorar el mal comportamiento, sólo porque el niño y niña están en cierta edad, ni debido a su 
sistema familiar, tal actividad implica que los adultos son importantes para ayudar a los niños a formar nuevos patrones de conducta. Los mayores pueden hacer algo con respecto al comportamiento infantil si sólo comprenden lo que les está pasando y se involucran más en mejorar sus relaciones. Que importante es que en el hogar los progenitores no sean desinteresados, sino que sean conscientes de que sus hijos e hijas los necesitan. La falta de interés, falta de atención a las necesidades básicas de un niño o un joven, que son: casa, comida, ropa, atención médica, asistencia, escuela, también tiene que ver con las necesidades de afecto, comunicación y vínculos fuertes entre papá, mamá, hijas e hijos.

\section{Factores pedagógicos}

\section{Factor escolar}

Para Beltrán (1995), los conflictos son casi inherentes en toda dinámica de trabajo, por tanto el docente no ha de descartar que la propia institución y la misma actividad escolar, pueden provocar desajustes conductuales; pero, en muchos casos, la escuela engendra nivel de ansiedad que constituye de manera decisiva el tipo de problema que genera, respecto a la disciplina. El excesivo número de estudiantes por clase, demasiado ruido, las características arquitectónicas del centro, como espacios reducidos o inapropiados, falta de instalación mínima, distribución física del aula y zonas de recreo y ocio, etc. En definitiva, todo ello tiene un prudente efecto sobre las actitudes de los estudiantes e influye en el rendimiento; por otra parte como es sabido en grupos de mala conducta se castigan fácilmente, pero en la escuela esto sucede de forma aún más acusada cuando se trata de conductas, que despiertan agresividad hacia los otros y hacia el docente.

Hay que estar sumamente convencido que los problemas de comportamiento disciplinario de los estudiantes no siempre inician en el hogar, sino por el contrario se dan en el colegio, debido a las condiciones del aula, a la falta de iluminación, al poco espacio en el que realizan sus actividades escolares, demasiados estudiantes atendidos por un solo docente, el calor, el ambiente aburrido y hostil, provocando en su mayoría la insatisfacción en el aula de clase y los resultados en su rendimiento escolar no son los más satisfactorios, y estamos conscientes que aunque el Ministerio de Educación mejore las condiciones de cada centro educativo este problema seguirá presente no a mayor escala, pero difícil de erradicar en la formación escolar.

El director del centro escolar "Niño Jesús" manifestó que las madres y padres, con los docentes deben tener una estrecha relación y comunicación en el centro educativo, porque son los actores principales en la educación. La formación de los estudiantes demanda un involucramiento de todos y no es tarea que concierne solamente al docente o la madre, cada uno debe asumir su papel y aportar en el aprendizaje en la 
escuela, estar pendientes del comportamiento de sus hijas e hijos y apoyarlos en sus tareas escolares para lograr un éxito y calidad en la enseñanza.

\section{Pedagogía inadecuada}

Se pudo observar que, otro determinante es la forma en que las docentes conducen el proceso de enseñanza-aprendizaje, situación que inconscientemente, la niñez aprovecha y presenta determinados comportamientos, como gritos, desesperación, inquietud y no muestra interés en escuchar las clases.

Lo anterior tiene estrecha relación con lo que refiere Martínez (2008), que la pedagogía no adecuada desmotiva y es determinante en la disciplina y comportamiento de la niñez en el salón de clases.

Es importante que cada miembro del personal docente haga una evaluación, revisión de la pedagogía que utiliza en la enseñanza y valora el mejoramiento de estrategias pedagógicas según las características del grupo. De esta forma garantizará el cumplimiento de su compromiso con la enseñanza.

\section{Influencia del factor económico en el comportamiento disciplinario de la niñez}

El personal docente en las entrevistas opinaron que la crisis económica está afectando a nivel mundial, y que la falta de recursos, la falta de servicios médicos, el ambiente de pobreza, la situación en la que vive la niñez es muy crítica, y afecta directamente su comportamiento, pero que siempre que las madres y padres de familia asuman su responsabilidad, con múltiples esfuerzos y sacrificios harán que sus hijos e hijas tengan su alimento diario, sus cuidados en lo concerniente a la salud y mejores condiciones de vida.

Es notorio que el comportamiento de los niños y las niñas, refiriéndonos a la disciplina en el Centro Escolar "Niño Jesús", no lo determinan los ingresos adquiridos por sus padres en los hogares. Se logró observar en general que tengan o no dinero, se comportan mal en el aula de clases y su rendimiento académico es bajo, por lo que el comportamiento de los estudiantes dependerá en gran forma del tipo de educación, valores y principios que los progenitores enseñen en sus hogares.

Las madres y padres de familia manifestaron que la pobreza que enfrentan en sus hogares influye en el comportamiento de la niñez en la escuela, ya que viven en hogares formados por madres solteras, que tienen que trabajar y el ingreso es muy bajo, debido a que se desempeñan como afanadoras y empleadas domésticas, cuyas condiciones y nivel de vida, carencia de dinero, servicios básicos, problemas en las condiciones de sus viviendas, hogares violentos, donde es obvia la situación social que viven o la escasez de todo lo necesario para vivir dignamente. 
Lo que coincide con Quintana (2001), al referir que cuando se habla de pobreza se suele pensar en falta de dinero, carencia de servicios básicos, problemas de vivienda, grupos marginales, violencia e inseguridad, entre otros. La pobreza es un fenómeno sobre el cual existe gran cantidad de definiciones, entre las que encontramos las siguientes: Una situación social que se caracteriza por la privación que tienen aquellos de algo necesario, deseado o de reconocido valor. Nivel de ingresos por debajo del cual es imposible obtener una alimentación adecuada desde el punto de vista de la nutrición y satisfacer las necesidades básicas no alimentarias. Carencia de bienes materiales considerados necesarios para el sustento de la vida, o también, la posesión muy temporal de ellos, en extremada escasez.

En las entrevistas aplicadas a las niñas y niños se encontró que es muy difícil su situación, refieren que desde muy pequeños se dedican a la venta de productos elaborados en casa, ejemplo: tortillas, horneados de maíz, nacatamales, entre otros; trabajan en el campo, cuidan a sus hermanos menores para aportar y contribuir al sostenimiento del hogar, y que lo hacen porque es la única forma para ayudar a sus familias a solventar las necesidades básicas del hogar, ya que sus progenitores no generan suficientes ingresos económicos, razón por la cual deben trabajar y luchar juntos para salir adelante.

Una pareja de hermanos expresaron:

Estamos cansados de realizar actividades, de trabajar, nos aburre esta vida, y después de ir a vender vamos a la escuela, pero vamos porque nuestros papás nos mandan y nos exigen, aunque nos cansamos y a veces no aprendemos bien las clases como mi papá y mi mamá quisieran, nos dicen que es importante que estudiemos para que tengamos un futuro mejor, que no seamos como ellos chapeando monte o echando tortillas.

Quintana (2001), expresa que cuando hablamos de trabajo infantil se refiere a cualquier actividad remunerada o no que los niños realizan y que significa un peligro nocivo para su desarrollo físico, social, moral y psicológico, dicha actividad representa en la mayoría de los casos un obstáculo para su pleno desarrollo. Los niños que viven en extrema pobreza, aprenden a sobrevivir desde pequeños, pues desde muy corta edad tienen que luchar por la vida, y esta vida hostil los hace ser una persona resentida. Este resentimiento lo lleva a cometer actos antisociales. Una de las características es la irritabilidad constante, lo que hace reñir con los demás por motivos insignificantes. En este medio se vale en cuanto se es macho (ya que no se puede valer por lo cultural, lo intelectual o lo económico) y así, el niño se convierte, desde pequeño en individuo altamente belicoso y agresivo. 
Debido a la pobreza y a la difícil situación económica que enfrentan la mayoría de los hogares de nuestro municipio siuneño, muchos niños y niñas se ven obligados a aportar a la manutención de sus hogares, realizando trabajos como vendedores en las calles; sin embargo, las madres y padres de familia tienen que luchar, inventar otras formas de sobrevivencia de manera que sus hijos tengan que dedicarse solamente a sus estudios y a obtener excelentes resultados en la escuela y no asumir responsabilidades que no les corresponde. De esta manera serán más felices, su formación será mejor y enfrentarán con facilidad los retos que se les presente en la vida.

\section{Papel de la Consejería Escolar en relación al comportamiento disciplinario}

Con este programa implementado en los centros escolares se cuenta con mecanismos para el desarrollo de los recursos humanos. Es una necesidad seguir capacitando a los docentes para que puedan enfrentar y encontrar las soluciones más apropiadas a los problemas que se presentan en la escuela, involucrando a la niñez en edad de escolaridad, padres y madres de familia del centro.

El objetivo principal de la Consejería Escolar es ayudar a resolver los diferentes problemas que enfrenta el estudiantado en el centro educativo. Este es el momento para aprovechar los conocimientos adquiridos por la consejería que orienta al escolar, y que le permite identificar las causas de dicho comportamiento, auxiliándose con material didáctico especializado en consejería, el cual puede utilizarse para brindar charlas, hacer presentación de videos educativos con sucesos reales, de tal forma que estos les ayuden en su desarrollo personal y escolar.

El director del centro escolar manifestó:

Mi función es tener acercamiento directo con los estudiantes, padres, madres de familia y docentes, el que me facilita un ambiente armónico entre todos y todas. El diálogo con las consejeras escolares y el involucramiento de los padres en el centro es esencial; para crear e implementar nuevas estrategias en conjunto y resolver los problemas que se presenta en el estudiantado. Actualmente, el desafío es mejorar su comportamiento, ya que muchas veces actúan de forma incorrecta, debido a la libertad que les brindan sus progenitores.

$\mathrm{Al}$ respecto, las consejeras dijeron que cuando tienen complicaciones serias, una de las acciones principales es comunicarles a los padres y madres de familia del comportamiento de los escolares, haciendo visitas continuas a los hogares, conversando con ellos sobre la situación de indisciplina, analizando bien el problema y proponiendo la solución en conjunto. 
Lo que coincide con De Trinidad (2005), que expresa que los consejeros escolares saben escuchar y pueden ayudar a los niños que atraviesan situaciones complicadas en sus vidas. Tienen una formación especial para ayudar a resolver problemas de la niñez en la edad escolar, a tomar decisiones y a defenderse solo.

Por su parte una madre de familia expresó:

La conducta de mi hijo es difícil y su cambio ha sido insuficiente, a pesar de los consejos y castigos que le doy. Han sido momentos difíciles, porque el director a diario me dice de la mala conducta de mi niño. A mí me da vergüenza, ya que él está grande para que me pongan quejas, yo no sé cómo orientarlo y ayudarle para que mejore su comportamiento. Yo trabajo desde las seis de la mañana hasta las siete u ocho de la noche, no dispongo del tiempo necesario para sentarme con él a dialogar de su actitud y de lo perjudicial que es para su futuro.

Un padre de familia expresó:

Los niños y las niñas necesitan consejos continuamente (ejemplos de lo que vivimos, de lo que vemos vivir). Hay ocasiones en los que yo castigo a mi hijo con algunas cosas que más le gustan. Es una tarea difícil, pero debemos incidir a diario para que todo sea mejor; sin embargo, tenemos que ser cuidadosos porque debemos de saber cómo corregir a nuestros hijos e hijas, aunque a veces nos excedemos. Cuando su indisciplina afecta el desempeño escolar es importante reunirse con el docente, el director o la consejera, para ver como se resuelve el problema, pero siempre hay que estar pendiente de ellos.

También las consejeras manifestaron que la mayor participación en los problemas del estudiantado los realizan las madres. Lo cual se convierte en un reto para ellas sensibilizar a los padres, para que participen en las actividades escolares de sus hijos en conjunto con los docentes, sólo así se podrán resolver situaciones de conflictos que surjan en dicho centro con mayor facilidad.

Tatar (2008), expresa que los consejeros escolares participan de la vida escolar en un grado considerable y desempeñan diversas tareas: asisten a los estudiantes con problemas de aprendizaje, o con dificultades sociales o emocionales, asumen un rol activo en la ubicación del estudiante por grado y sección, refieren a los estudiantes a los profesionales externos, son consultores de padres y docentes, y también apoyan a los directores en temas organizacionales que tendrán impacto en las vidas de los estudiantes. 


\section{EDUCACIÓN}

En la entrevista realizada a la consejera escolar esta expresó que en el centro educativo se evidencian diferentes tipos de comportamientos, entre ellos se encuentran niños y niñas muy pasivos, otros hiperactivos, maleducados, irrespetuosos e irresponsables, razón por la cual conociendo el panorama o el contexto de la situación real de la escuela, se les ayuda para que haya un cambio en su forma de comportarse a través del seguimiento continuo para superar estos problemas.

En las diferentes secciones hay grupos con indisciplina, más en los niños que son los que comienzan a descontrolar el salón cuando la docente está impartiendo su clase, no prestan atención, lo que dificulta un buen aprendizaje y por ende obtienen malas calificaciones. Algunos niños, una vez que finalizan sus tareas escolares empiezan a andar molestando a los demás, hacen el alboroto, otros tienen un mal comportamiento en su forma de ser, porque reciben una mala formación en sus hogares o no ponen en práctica sus valores y principios morales. Usan un mal vocabulario en el aula de clase, y demuestran rebeldía ante sus docentes, director y consejera escolar.

Al respecto De Trinidad (2005), expresa que eso no significa que el consejero tenga una varita mágica que hará desaparecer el problema. Pero sí que te ayudará a afrontarlo. Afrontar es una palabra que es muy importante conocer. A veces, los niños $\mathrm{y}$ los adultos tienen problemas complicados. Afrontar significa intentar manejar esos problemas y mejorar las cosas. Están dispuesto a escuchar y quiere que la experiencia escolar sea lo más positiva posible. La función de un consejero es tomarse en serio tu problema y ayudarte a encontrar una solución. Al consejero también le interesa ayudarte a aprender al máximo en clase, que seas un miembro activo de la comunidad escolar y que tengas una visión positiva sobre el medio escolar.

Si bien es cierto, en el proceso educativo existen diferentes formas de equilibrar el comportamiento de la niñez en la escolaridad, no hay que olvidar que esta tarea está encomendada a los docentes que laboran en determinados centros de estudios, pero que también la educación es responsabilidad de las madres y padres de familia y que ahora se cuenta con el apoyo de la Consejería Escolar, la cual realiza un estupendo trabajo. Tratar de ayudar a los estudiantes a que mejoren la conducta y su comportamiento educativo es un reto primordial para la Consejería Escolar.

Es un proceso de acompañamiento por etapas y su duración dependerá del estudiante, esto no quiere decir que el resultado será inmediato, al contrario, tomará un lapso necesario para reconocer errores y faltas que frecuentemente comete sin reparo alguno, y que sin ayuda de otras personas no podrá corregir. Estas acciones desarrolladas por la Consejería Escolar en coordinación con los docentes, están orientadas con un solo propósito, ayudar a cada estudiante que sea razonable y que esté consciente del bien que le hará tomar dichas sugerencias y poder aplicarlas en su entorno educativo, familiar y social. 


\section{Lista de referencias}

Beltrán, J. (1995). Psicología de la educación. Primera edición. Barcelona, España: Pearson Educación.

De Trinidad, B. (2005). Género - violencia intrafamiliar e ITS. Managua, Nicaragua: URACCAN.

Quintana, M. (2001). Educación y trabajo infantil, principales problemas. Managua, Nicaragua: UNICEF, Vol. III.

Rourat, J. (2001). Psicología de la pubertad, los efectos del divorcio en los hijos. Editorial Luis Miracles, S. A. Barcelona.

\section{Revistas}

Cáceres, C. (2007). Revista despertad: Los padres deben poner atención a sus hijos, vol. 88, no. 6, p. 4.

Martínez C. (2008) Timonel Revista digital ISSN 1576 - 866X. Cádiz España.

Tatar, M. (2008). Revista de psicología: Contexto cultural y consejería escolar: sensibilidad cultural y defensa de la justicia social. Vol. XXVI (1). Israel: Hebrew University of Jerusalem, Israel. 